\title{
Predicting Commitment to Wed Among Hispanic and Anglo Partners
}

By: Adriana J. Umana-Taylor and Mark A. Fine

Umana, A., \& Fine, M. A. (2003). Predictors of commitment to wed among Hispanic and Anglo partners. Journal of Marriage and Family, 65(1), 117-129. DOI: 10.1111/j.1741$\underline{\text { 3737.2003.00117.x }}$

Made available courtesy of Wiley-Blackwell. The definitive version is available at onlinelibrary.wiley.com.

\begin{abstract}
***Reprinted with permission. No further reproduction is authorized without written permission from Wiley-Blackwell. This version of the document is not the version of record. Figures and/or pictures may be missing from this format of the document. ***
\end{abstract}

\begin{abstract}
:
Ethnic differences in commitment to wed were examined between 46 Hispanics (27 women, 19 men) and 160 Anglos (84 women, 76 men). Although limited by sample sizes, findings indicated that Hispanics and Anglos did not differ, on average, on measures of attitudes toward marriage, perceived family influence, commitment to wed, belongingness, and trust. Hierarchical regression analyses revealed that, after controlling for age and income, attitudes toward marriage, perceived family support, and trust predicted commitment to wed for women, whereas only perceived family support emerged as a predictor among men. Finally, although no ethnic differences emerged for men, the degree to which trust, perceived family support, and attitudes toward marriage predicted commitment to wed for women varied by ethnicity.
\end{abstract}

Keywords: marriage | commitment | dating | relationships | Hispanics

\section{Article:}

Commitment can be defined as partners' perceptions of the likelihood that their relationship will continue over the long run (Surra \& Hughes, 1997). Given that approximately 62\% of women in the United States aged 15-44 have ever been married (Bramlett \& Mosher, 2001) and that the mean age at first marriage is 25 for women and 27 for men (Clarke, 1995), it is critical to examine factors influencing the decisions to commit to wed among the young adult population. Studies of commitment to romantic relationships have been conducted almost entirely with middle-class Anglo respondents and thus may not generalize beyond this population. Cultural variations in the socialization of people from different ethnic and racial backgrounds are likely to influence not only how committed people are to their relationships, but also what determines their commitment to marry. The primary questions addressed in this study were: (a) Do Hispanics and Anglos differ on commitment-related relationship and individual variables? and 
(b) Are there ethnic differences in the degree to which love, trust, attitudes toward marriage, and perceived family support influence commitment to wed for Anglos and Hispanics?

Researchers who have examined commitment in relationships have focused on global commitment rather than on commitment to wed (Surra, Hughes, \& Jacquet, 1999). Whereas global commitment concerns individuals' beliefs about whether their relationship will last over the long run, commitment to wed concerns partners' conceptions that they will form and maintain a marriage to a partner for the foreseeable future (Surra et al., in press). It is important to take into account differences between commitment to wed and global commitment when studying individuals from different cultural backgrounds. In cultures where the institution of marriage is highly valued, commitment to wed and global commitment might be more distinct constructs than in cultures where marriage is less highly valued.

Commitment was conceptualized in this study according to a categorization proposed by Johnson (1991), who has suggested that individuals in a relationship experience moral, personal, and structural commitment. Moral commitment is the extent to which one's own value system and sense of right and wrong influence the decision to stay in a relationship; personal commitment involves the extent to which one wants to continue in a relationship as a result of one's attitudes about and identity with the relationship or the partner; and structural commitment involves the extent to which environmental constraints make it difficult to leave a relationship.

Researchers who have studied the predictors of commitment in romantic relationships have emphasized the components of personal commitment (Hughes \& Surra, 1998). Personal commitment includes constructs such as love, satisfaction, attachment to the partner, closeness to the partner, and sense of belongingness (cf. Hughes \& Surra; Johnson, 1991; Rusbult, 1983). Theorists have maintained that elements of personal commitment are the most powerful predictors of commitment in romantic relationships. It is our premise, however, that the importance of personal aspects of commitment may vary by ethnic background.

To theoretically ground our exploration of Hispanic-Anglo differences in the determinants of commitment, we made use of an individualistic versus collectivistic worldview dimension. Individualism involves the tendency to look out for the needs of oneself or one's immediate family, whereas collectivism involves the integration of individuals into cohesive groups that demand unquestioning loyalty among group members (Hofstede, 1991). Along these lines, individualistic societies emphasize autonomy, emotional independence, and self-directed 
initiative, whereas collectivistic societies emphasize group identity, emotional dependence, and group decision making (Hofstede, 1980). Specific to this study, previous research indicates that Hispanic cultures tend to be characterized by collectivistic ideologies, whereas English-speaking cultures tend to adhere to individualistic values (Falicov, 2001; Freeberg \& Stein, 1996; Murphy, 1998). The collectivistic orientation of Hispanic cultures coincides well with the structural and moral components of commitment, whereas the individualistic ideals demonstrated by many European cultures lend themselves well to aspects of Johnson's (1991) personal commitment. As a result, it is not surprising that work on commitment to marital relationships, which has been conducted primarily with White, middle-class respondents, has tended to focus on the personal aspects of commitment. It is important to consider that individuals whose ethnic backgrounds are associated with a collectivistic orientation may adhere more to structural or moral aspects of commitment than to personal aspects of commitment.

For example, belongingness and trust, which can be viewed as aspects of personal commitment, have been found to be associated with commitment for Anglos, but they may be less important determinants of commitment among Hispanics. By contrast, Johnson's (1991) concepts of moral and structural commitment seem to mesh well with the family socialization of Hispanics. According to Johnson, moral commitment develops from three sources: (a) a belief in the value of consistency (e.g., standing by one's choices), (b) values regarding the stability of particular types of relationships, and (c) a personal contractual obligation to a partner. Concerning the second source of moral commitment, Johnson explained that in American culture it is acceptable to end a dating relationship but less acceptable to end a marriage. For Hispanics, however, ending a dating relationship may not be as acceptable as it is for Anglos, because Hispanics consistently report a high level of family commitment (Hurtado, 1995). Because of this strong belief in the institution of family, Hispanics may be less likely to date someone casually or to continue to date someone whom they are not committed to marrying.

Johnson's (1991) structural commitment may also play a large role in the relationships of Hispanics. Social reaction, a source of structural commitment, refers to the pressure from the social network to remain in the relationship. Among Hispanics, because of their close attachments to family (discussed later), social reactions from family members could affect the decisions they make in relationships. Because of a collectivistic orientation, Hispanics may be more influenced by what their family members think than by personal commitments that they may have to the relationship. In the paragraphs that follow, we show how two sets of values characteristic of Hispanic cultures (i.e., traditionalism and familism) might affect relationships. 
Research indicates that Hispanics not only hold traditional views and behaviors regarding family life (Eberstein \& Frisbie, 1976; Staples \& Mirande, 1980) but also manifest traditional attitudes in some of their dating behaviors. For example, Golding and Baezconde-Garbanati (1990) found that Mexican Americans were less likely to cohabit than were non-Hispanic Whites, and several researchers have found that cohabiting individuals hold more nontraditional family values (Demaris \& MacDonald, 1993; Nock, 1995). Traditional values can also be assessed by examining individuals' attitudes toward marriage. Individuals with more traditional attitudes toward marriage may be more committed to relationships that could result in marriage.

In addition, the often-made generalization that Hispanics have more familistic values than Anglos is supported by an abundance of research (see Vega, 1990, for a review). Researchers have found that Hispanics, relative to Anglos, have more tightly integrated local extended families (Golding \& Baezconde-Garbanati, 1990; Keefe, 1984), are more likely to be married (Golding \& Baezconde-Garbanati; Roberts \& Roberts, 1982), and are more likely to confide in family members than in friends (Bryant, Surra, \& Swim, 1996; Keefe, Padilla, \& Carlos, 1979). Finally, Hispanics have closer attachments to their family of origin, more reliance on kin, a larger number of relatives who live nearby, and a higher frequency of kin visiting than Anglos (Keefe, 1984; Mindel, 1980). In line with these ideas, we hypothesized that Hispanic partners would hold more positive attitudes toward marriage, would be more committed to marrying their dating partners, and would report that kin and family members influenced the decisions they made concerning their relationships more than would Anglo partners.

The present study explored the possibility that individuals from different cultural backgrounds may give weight to different considerations when evaluating their level of commitment to their relationship. If, because of their familistic orientation, Hispanics hold more traditional attitudes toward marriage than do Anglos, they may be more likely to take romantic relationships seriously, not become romantically involved with individuals whose values appear different from their own, and exhibit a consistent commitment to making their relationships work. Along those same lines, if, because of their collectivistic orientation, Hispanics have a close attachment to their family of origin and rely strongly on family and kin, the support of their family members for their dating relationships may profoundly influence their level of commitment to their relationships. Accordingly, we hypothesized that aspects of structural commitment (perceived familial support) and moral commitment (attitudes toward marriage) would be more strongly related to commitment to wed for Hispanic partners than for Anglo partners. Furthermore, in line with previous research, we hypothesized that aspects of personal commitment, namely belongingness and trust, would be more strongly related to commitment to wed for Anglo partners than for Hispanic partners. Finally, all analyses were conducted controlling for age and income, which have been found to be related to relationship outcomes in previous work (e.g., 
Booth \& Edwards, 1985; Bumpass, Martin, \& Sweet, 1991; White \& Rogers, 2000). It is desirable to control for age and income because these variables are part of the social context in which individuals' relationships are embedded and thus may influence commitment processes.

\section{Method}

\section{Sample}

The current study consisted of 46 Hispanics and 160 Anglos who were part of a larger study that longitudinally examined commitment to wed among dating partners. Respondents were recruited from a metropolitan area in the Southwest by means of a random-digit-dialing procedure. To be eligible to participate, respondents had to be between the ages of 19 and 35 years, English speaking, single and never married, and currently dating someone of the opposite sex. The dating partners of the respondents who initially agreed to participate were contacted and also asked to participate. Of all eligible couples contacted, 27\% agreed to participate. A total of 464 individuals, or 232 couples, formed the sample for the larger study. Of the 464 respondents, $68 \%$ were Anglo, 18\% were Hispanic, 8\% were African American, 6\% were Asian or Pacific Islander, and less than $1 \%$ were Native American. For the purpose of this article, only Anglo and Hispanic respondents were included in the analyses. Although every person in the sample had a partner who was also participating in the study, Anglo participants were more likely to be dating an Anglo partner than Hispanics were likely to be dating a Hispanic partner. This is likely a result of the population characteristics of the area, in which Anglos make up 61\% of the population and Hispanics make up only $26 \%$ of the population. Because of this, of the sample of Anglo and Hispanic respondents included in the current study, $65 \%$ and 27\%, respectively, were dating people of the same ethnicity as themselves. Finally, because Hispanics of Mexican origin constitute $90 \%$ of the Hispanic population in the state where the data were gathered (U.S. Census Bureau, 1990), it is likely that the majority of the Hispanics in the current study were of Mexican origin.

To be included in the current analyses, respondents had to remain in the study through all three phases and have complete data on all measures included in the analyses. Of the 84 Hispanics and 317 Anglos who participated in the larger study, 46 Hispanics and 160 Anglos had complete data for all variables assessed in the present study. The mean age of the Hispanic respondents was 23.2 years for men and 22.9 years for women, whereas the mean for Anglos was 25.6 years for men and 23.3 years for women. These figures are comparable to the figures for the U.S. population's mean age at first marriage (i.e., 27 for men and 25 for women). Thus, it is likely that the respondents in our sample were at an age where they were experiencing the processes that would lead to marriage, making commitment to wed an appropriate topic to study with this 
population. Finally, mean years of education were 15.0 for Hispanic men, 14.7 for Hispanic women, 15.2 for Anglo men, and 14.9 for Anglo women.

Hispanic respondents who had complete data on all variables examined in this study did not differ significantly from their counterparts who did not have complete data with regard to income, education, and age. Conversely, Anglo respondents who had complete data were significantly older $(\mathrm{M}=24.34$ years, $\mathrm{SD}=3.8$, vs. $\mathrm{M}=23.11$ years, $\mathrm{SD}=3.3 ; \mathrm{p}<.01)$ and reported significantly higher levels of education $(\mathrm{M}=15.03$ years, $\mathrm{SD}=1.9$, vs. $\mathrm{M}=14.48$ years, $\mathrm{SD}=1.6 ; \mathrm{p}<.05$ ) than their counterparts who did not have complete data on all study variables.

\section{Procedure}

This study consisted of three phases of face-to-face interviews conducted over a 9-month period. Respondents were interviewed approximately once a month and were compensated $\$ 20$ for completing the first long interview, $\$ 5$ for completing each of the seven short monthly interviews, and $\$ 20$ for completing the final long interview. Coupled partners were interviewed separately, and all respondents were encouraged to complete all interviews regardless of the status of their relationships or the continued participation of their partner.

In Phase 1, respondents completed a series of questionnaires, answered some questions orally, constructed a graph of commitment to marry their current dating partners, and answered questions about their relationships on a game board using a set of cards. Phase 1 interviews lasted approximately 1.5 to 2 hours. To identify ethnicity, individuals were asked: (1) "What is your race?" and (2) "Some people have a particular nationality or ethnicity in their family background that they identify with. Is this true of you?" If respondents answered yes to the second question, they were asked, "What is it?" Respondents who answered Hispanic or any other Hispanic subgroup (e.g., Colombian, Mexican) to the first question or to the second part of the second question were classified as Hispanic. Respondents who answered White or Caucasian to the first question and did not indicate a Hispanic affiliation in the second question were classified as Anglo. For the purpose of the present analyses, the social background questions and two separate questionnaires assessing belongingness and trust were used from Phase 1.

In Phase 2, which consisted of seven interviews, respondents were interviewed once a month to update changes in their relationships. These interviews lasted 15 to 20 minutes. One to five questionnaires were completed at each interview. Graphs of commitment were drawn at each of 
the interviews in this phase. For this article, only a questionnaire assessing attitudes toward marriage was used from Phase 2.

The final interview, Phase 3, was similar in length and procedure to the initial interview. Once again, the graph of commitment was drawn, and respondents completed questionnaires. A majority of the questionnaires administered at Phase 1 were readministered at Phase 3 . The graph of commitment and one questionnaire that assessed the importance of opinions and beliefs of friends, relatives, peers, and dating partners were used from this phase.

The graph of commitment to marry mapped the course of individuals' commitment to marrying their current dating partner over the course of their relationship. The procedure was originally introduced by Huston, Surra, Fitzgerald, and Cate (1981). Commitment to marry was operationalized as percentage chance of marriage. Chance of marriage was recorded on the yaxis of a blank graph and ranged in 5\% increments from 0 to $100 \%$. The chance of marriage at $100 \%$ indicated that respondents were certain they would marry their partner, and $0 \%$ indicated that respondents had never thought about marrying their partner, even briefly, or that respondents were certain they would never marry their partner. Time in months was recorded on the x-axis. At Phase 3, the graph was drawn retrospectively from the date that the relationship began until the date of the Phase 3 interview. Chance of marriage was defined in the following manner:

There may have been times when you have thought, with different degrees of certainty, about the possibility of marrying [dating partner]. These thoughts are based on your ideas about marrying [dating partner] and on what you think have been [dating partner's] thoughts about marrying you. Taking both of these things into consideration, we will graph how the chance of marrying [dating partner] has changed from the time you met [dating partner] until today.

The respondents gave the interviewers a list of key events that had taken place over the course of the relationship, and the interviewers wrote the events on the x-axis of the graph. The date that the relationship began and the date of the interview were marked on the x-axis of the graph, and the respondents were asked to tell the interviewer what the chance of marriage was at the present time (i.e., the date of the interview). Then the interviewers asked what the chance of marriage was at the beginning of the relationship. Once those two points were marked on the graph, the interviewers asked the respondents when they first noticed that the chance of marriage was different from what it had been at the beginning of the relationship. After the respondents gave a 
date for the first increase or decrease in chance of marriage, the interviewers asked the respondents what the chance of marriage was on that date. After drawing a dot on the graph for the appropriate time and chance of marriage value, the interviewers asked the respondents what the line connecting the two dots should look like, and proceeded to draw the line. The line drawn to connect the two points constitutes a turning point in the commitment graph. After the line was drawn, the interviewers asked the respondents to tell them, in as specific terms as possible, what happened from [date] to [date] to make the chance of marriage [increase or decrease][a certain] percent. After the respondents answered this question, the interviewers asked if there were anything else that happened to change the chance of marriage. When the respondents answered no to this probe, the interviewers began the procedure again and asked the respondents when they noticed that the chance of marriage was different from the previous chance of marriage. This sequence was carried out throughout the entire length of the relationship until the date of the interview was reached on the graph.

\section{Measurement}

\section{Attitudes toward marriage}

A 14-item scale adapted by Kinnaird and Gerrard (1986) assessed attitudes toward marriage. This questionnaire was administered during the fifth interview of Phase 2 or during the sixth interview if respondents missed their fifth interview. The items (e.g., "How difficult would it be for you to adjust to married life?”) were scored on a five-point Likert scale, with end points of not at all difficult (1) and very difficult (5). Higher scores indicated more traditional attitudes toward marriage. Cronbach's alphas were .89 for Hispanics and .85 for Anglos.

\section{Commitment to wed}

The graph of commitment to marry was drawn during every interview, regardless of whether respondents' relationships had ended. Only the graph drawn during Phase 3 was used for this study. Two indicators were derived from the graph to measure commitment to wed. The first indicator, proportion of downturns, was computed by adding the number of turning points in which the chance of marriage decreased and dividing that number by the total number of turning points in the graph. The second indicator, mean absolute slope, was computed by taking the absolute value of the percentage change in the chance of marriage for a turning point and dividing it by the number of weeks in the turning point. The mean absolute slope was computed by summing the absolute values of the slope for each turning point and dividing that by the total number of turning points in the graph. This variable reflects the mean rate of change per turning point in the graph. 
Individuals who have a high proportion of downturns in their graph are considered to be less committed to marrying their dating partners, because they have a relatively large number of decreases in commitment. Similarly, a large value for the mean absolute slope indicates a high degree of change in commitment to wed over the length of the relationship. High commitment to a relationship is indicated by lack of variability in the level of commitment to a relationship over time (Kelley, 1983; Surra et al., 1999). Low variability in the commitment level indicates that there is less turbulence or dramatic change in commitment over time. Therefore, the lower the mean absolute slope, the higher the commitment to wed. In this study, proportion of downturns and mean absolute slope were not significantly correlated (for Anglos, $r=.03$; for Hispanics, $r=$ .08), and thus all analyses were performed twice, once with mean absolute slope as the dependent variable and once with proportion of downturns as the dependent variable.

\section{Characteristics of the social network}

An instrument designed for Phase 3 by Bryant et al. (1996) was used to assess the involvement in and importance of social networks in partners' relationships. Respondents were told, "Identify up to 10 people whose opinions of your personal life are most important to you.” Then, for all people listed, they identified the sex, race, and relationship to the respondent and answered several questions. Only three questions from this instrument were included in the present analyses. Two variables were derived using those three 7-point Likert-scaled items. The first variable, perceived family influence, was computed using the question, "If [individual named] let me know that I should stop dating my current dating partner, I would seriously consider his/her advice,” with end points of strongly disagree (1) and strongly agree (7). This variable was computed by summing all of the scores on this item for any individual listed who was a family member of the respondent and dividing by the number of family members listed. Family members included mothers, fathers, siblings, aunts, uncles, grandparents, and other kin. Perceived family influence was used to assess the degree to which respondents perceived their family members as influencing them when making decisions about their dating relationship.

Because the question for perceived family influence was hypothetical, a second measure was computed to assess what actual support for their relationships respondents felt they received from family members. The second measure, perceived family support, was computed using the same procedure as just discussed. It assessed how much encouragement or approval of their dating relationship respondents perceived that they received from family members. For this measure, two questions were used: "To what degree do you think [individual named] disapproves or approves of your current dating relationship?” with end points of very much disapproves (1) and very much approves (7), and "Overall, how much actual discouragement or 
encouragement do you get from [individual named] to continue to date your current dating partner?” with end points of discouraged a great deal (1) and encouraged a great deal (7). Cronbach's alphas for this measure were .91 for Hispanics and .74 for Anglos.

\section{Belongingness}

The belongingness subscale, derived from Braiker and Kelley's (1979) scale of relationship dimensions, was administered during Phase 1 of the study. A factor analysis was conducted with the entire sample (Jacquet \& Surra, 2001) and indicated that 9 of the 10 items in the original love scale (e.g., “To what extent do you have a sense of 'belonging' with your partner?”) loaded on one factor. Two additional items (i.e., "To what extent do you try to change your own behavior to help solve certain problems between you and your partner?" and "To what extent do you reveal or disclose very intimate facts about yourself to your partner?”), derived from Braiker and Kelley's maintenance subscale, also loaded on this same factor. The 11-item factor was termed belongingness to distinguish it from other measures of love employed in the larger study. Factor scores were used to test the hypotheses. Cronbach's alphas on the belongingness scale were .87 for Hispanics and .88 for Anglos.

\section{Trust}

The eight-item Dyadic Trust Scale (Larzalere \& Huston, 1980) was used to measure trust. A factor analysis was performed with data from all respondents in the larger study, and two factors were derived (Jacquet \& Surra, 2001). The factor scores from the analysis formed the two trust variables, trust in a benevolent partner and trust in an honest partner, which were used to test the hypotheses. Higher scores indicated higher levels of trust in the benevolence or the honesty of the partner. The trust in an honest partner measure yielded Cronbach's alphas of .78 for Hispanics and .84 for Anglos. The trust in a benevolent partner measure yielded Cronbach's alphas of .74 for Hispanics and .70 for Anglos.

\section{Results}

We examined our hypotheses using analysis of covariance (ANCOVA) and hierarchical regression analyses. For one of the dependent variables - proportion of downturns — arc sine transformations were performed.

Data for this study were gathered from both members of couples. Thus, we had to consider the possible nonindependence of reports from coupled partners in our approach to analyses (cf. 
Kenny, 1988). Thus, we ran our analyses separately for men and women to control for the nonindependence of the data. Because of the limited sample size for Hispanic men, their findings should be interpreted with caution.

\section{Mean differences between hispanics and anglos}

We predicted that Hispanics and Anglos would differ significantly in their attitudes toward marriage, commitment to marrying their dating partners, and the degree to which their families influenced their dating relationships. A series of ANCOVAs was conducted separately for men and women, with ethnicity as the independent variable and age and income as covariates. Results indicated that, on average, Hispanics and Anglos in this sample did not differ on these variables (results available upon request).

\section{Differences in predictors of commitment to wed for hispanics and anglos}

Our second research question pertained to whether there were ethnic differences in the degree to which certain variables predicted commitment to wed for Hispanics and Anglos. We hypothesized that attitudes toward marriage and perceived family support would predict commitment to wed more strongly for Hispanics than for Anglos and that belongingness, trust in an honest partner, and trust in a benevolent partner would predict commitment to wed more strongly for Anglos than for Hispanics.

To examine ethnic group differences in the predictors of commitment to wed, we used hierarchical regression analyses with interaction terms, in which ethnicity was dummy coded as 0 for Anglos and 1 for Hispanics. In addition, all analyses were conducted controlling for income and age. For each dependent variable, a three-step hierarchical regression analysis was computed. In Step 1, income and age were entered into the equation; in Step 2, ethnicity and the five hypothesized predictor variables were entered; finally, in Step 3, five interaction terms (i.e., the product of ethnicity and each predictor variable) were entered. When Step 3 produced a significant change in R2, there was a significant interaction between ethnicity and the independent variable in predicting commitment.

As previously mentioned, all analyses were conducted separately by sex to control for the nonindependence of couple data. In addition, separate regression models were run for each of the dependent variables (i.e., mean absolute slope and proportion of downturns). No significant findings emerged for men or women in the analyses in which mean absolute slope was the 
dependent variable (results available upon request); thus, the results that follow represent analyses in which proportion of downturns was examined as the dependent variable (see Table 1 for correlations between the independent and dependent variables).

\section{TABLE 1 HAS BEEN OMITTED FROM THIS FORMATTED DOCUMENT}

Results for women suggested that, after controlling for income and age, $24 \%$ of the variance in proportion of downturns was explained by the combination of the five independent variables and ethnicity (see Table 2). Specifically, in the context of the other independent variables, attitudes toward marriage independently contributed to the variability in proportion of downturns. Although only attitudes toward marriage emerged as an independent contributor in the multivariate context, the bivariate correlations (see Table 1) indicated that proportion of downturns was significantly and inversely related to attitudes toward marriage, perceived family support, and belongingness for both Hispanic and Anglo women; to trust in a benevolent partner for Hispanic women only; and to trust in an honest partner for Anglo women only.

\section{TABLE 2 HAS BEEN OMITTED FROM THIS FORMATTED DOCUMENT}

In addition, the interaction terms that were entered in Step 3 of the hierarchical model produced a significant $10 \%$ change in R2. Specifically, significant interactions emerged for perceived family support and trust in a benevolent partner, and the interaction involving attitudes toward marriage approached significance.

To follow up, partial correlation coefficients (controlling for the other independent variables, income, and age) between the independent and dependent variables were compared for Hispanic and Anglo women. Results indicated that attitudes toward marriage (partial $r$ for Hispanic women $=-.64$; partial $\mathrm{r}$ for Anglo women $=-.13$ ) and trust in a benevolent partner (partial $\mathrm{r}$ for Hispanic women $=-.48$; partial $\mathrm{r}$ for Anglo women $=-.01$ ) were more strongly associated with proportion of downturns for Hispanic women than for Anglo women, after holding constant all other independent variables. Although trust in a benevolent partner and attitudes toward marriage were positively related to commitment to wed among both Hispanic and Anglo women, the relationship was stronger for Hispanic women. 
Finally, the direction of the relationship between perceived family support and proportion of downturns was different for Hispanic and Anglo women (partial r for Hispanic women = .31; partial $\mathrm{r}$ for Anglo women $=-.23$ ), indicating that higher levels of perceived family support were associated with fewer downturns for Anglo women but with more downturns for Hispanic women. Thus, controlling for all other independent variables, as Hispanic women reported more downturns in their commitment to marry (i.e., less commitment), they also reported higher levels of perceived family support. Conversely, the more Anglo women perceived that their families supported their relationships, the more committed they were to marrying their partner.

Results for men indicated that, after controlling for age and income, 22\% of the variance in proportion of downturns was explained by the combination of the variables (R2 after Step $2=$ $.27, \mathrm{~F}=3.88, \mathrm{p}<.0001$ ). The only significant independent contributor to the model, however, was perceived family support $(\beta=-.40, \mathrm{p}<.0001)$. The more support that men perceived themselves as receiving from their families regarding their dating relationships, the lower was their proportion of downturns. Unlike the results for women, no significant interactions emerged for men, indicating no ethnic differences in the degree to which certain variables predicted commitment.

\section{Discussion}

In this study, we examined whether Hispanics and Anglos differed on various relationshiprelated variables as well as whether structural, personal, or moral aspects of commitment would predict commitment to wed more strongly for one ethnic group than for the other. Methodologically, this study improved upon previous studies on dating relationships by (a) studying Hispanic individuals, (b) examining both individual- and relationship-level predictors of commitment (i.e., attitudes toward marriage as well as belongingness and trust), and (c) studying a more specific type of commitment (i.e., commitment to wed rather than global commitment). With these advances, we found that Hispanics and Anglos, on average, did not differ with regard to attitudes toward marriage, commitment to wed, or perceived family influence on their relationships. However, the degree to which certain variables predicted commitment to wed varied by ethnicity for women. Our findings are presented as exploratory and should not be considered to be generalizable to all Hispanics; rather, we hope that this study will contribute to the design of future studies on premarital commitment with Hispanic populations.

\section{Mean differences between hispanics and anglos on commitment-related variables}


Although we expected Hispanics and Anglos to differ on attitudes toward marriage, proportion of downturns, mean absolute slope, and perceived family influence, we found no significant mean differences between them. One possible explanation is that the Hispanics in this study may be less traditional and more acculturated than the Hispanics who have previously been studied. In fact, the Hispanics in our study were younger (i.e., mean age was 23) than the Hispanics examined in the studies that we reviewed (i.e., mean ages ranged from 38 to 43) (see Golding \& Baezconde-Garbanati, 1990; Keefe et al., 1979). Furthermore, 55\% of the Hispanics in this study were dating individuals who were not of Hispanic descent, which might indicate that they were not as traditional as other Hispanics who did not date interethnically. Administering a measure of acculturation would have allowed us to determine if similarities between the Hispanics and Anglos in our study resulted from the acculturation of Hispanics, given that as level of acculturation increases, Hispanics become more similar in attitudes and behaviors to Anglos (Domino \& Acosta, 1987; Griffith \& Villavicencio, 1985).

\section{Predictors of commitment to wed among women}

Using hierarchical regression analyses, we found that attitudes toward marriage predicted commitment to wed among Hispanic and Anglo women and that perceived family support approached significance as an independent predictor. For both Hispanic and Anglo women, as attitudes toward marriage became more positive, proportion of downturns decreased, indicating a greater commitment to wed. In line with our hypotheses, this relationship was stronger for Hispanic women than for Anglo women. Compared to Anglo women, the commitment to wed of Hispanic women changed to a greater degree (greater variability in chance of marriage reflects less overall commitment) to the extent that their attitudes toward marriage were less positive. This suggests that aspects of moral commitment may have stronger effects on commitment to wed for Hispanic women than for Anglo women, perhaps because of the close relationship between attitudes toward marriage and Latino cultures' familistic ideals.

Contrary to our expectations, in the multivariate context, we found that commitment to wed was positively related to family support for Anglo women, but negatively related to family support for Hispanic women. Thus, when all other independent variables were considered, the more familial support of the relationship that Hispanic women reported, the lower their commitment to wed their partner. One possible explanation is that perhaps when Hispanic family members are supportive of a dating relationship, they may get more involved in the relationship than do Anglo family members. Although they may intend to be supportive, they may actually cause strife in the relationship. When families are not perceived as supportive, perhaps there is little involvement on the part of family members, and therefore the relationship takes its own course. Thus, in Hispanic families, family support may be associated with increased family involvement 
and, in turn, less freedom for the couple, which could lead to more downturns in the relationship. For Anglo women, when family members are supportive of the relationship, family members may not be overinvolved. Thus, even though they may support the relationship, they may not interfere; the families of Anglo women may provide a more distant type of support that allows the relationship to develop on its own. These explanations are necessarily speculative, because the findings in the multivariate and bivariate contexts differed. Whereas the bivariate correlations indicate that, as expected, perceived family support and proportion of downturns were negatively related, in the multivariate context, when all other independent variables were controlled, this relationship became positive. Thus, the positive relationship should only be interpreted in the context of the variables being controlled.

Finally, one additional ethnic difference emerged that was contrary to our hypothesis. Commitment to wed was significantly more strongly related to trust in a benevolent partner for Hispanic women than for Anglo women. The more Hispanic women trusted the benevolence of their partner, the lower their proportion of downturns; this relationship was not significant for Anglo women. It is difficult to interpret this finding, given that we are not aware of any research in which trust and commitment to wed were examined among Hispanic women. Future work should examine whether this aspect of personal commitment is more influential than structural and moral aspects of commitment on the relationships of Hispanic women. Perhaps qualitative techniques, such as asking Hispanic women about the meanings that they attach to commitment to wed, can guide future work on this topic.

Taken together, our findings suggest that aspects of personal, moral, and structural commitment are influential predictors of commitment to wed for both ethnic groups, although some ethnic differences emerged with regard to the direction and the degree of the relationships.

\section{Predictors of commitment to wed among men}

Results for men indicated that perceived family support significantly predicted commitment to wed for both Hispanics and Anglos, with greater perceived familial support being associated with a lower proportion of downturns. However, unlike the findings for women, there were no ethnic differences in the predictors of commitment for men. Results for men, especially the absence of significant differences, should be interpreted with caution, because the sample size for Hispanic men was small. In fact, the bivariate analyses suggest that the correlations between proportion of downturns and belongingness and trust in an honest partner were different in expected ways between Hispanic and Anglo men. Thus, it is likely that low statistical power contributed to our inability to detect differences for men in predictors of commitment to wed. 


\section{Differences between the two measures of commitment}

Two indices of commitment were used as dependent variables (i.e., proportion of downturns and mean absolute slope) in this study. In the multiple regression analyses, the only significant results occurred for proportion of downturns. One explanation for our lack of significant findings pertaining to mean absolute slope is that we did not assess variables that have been related to this variable in previous studies (Surra, Arrizi, \& Asmussen, 1988; Surra \& Hughes, 1997). Another possible explanation is that, although both dependent variables assess change in commitment, they measure different types of change. Proportion of downturns measures only negative changes in commitment, because it measures, relative to the total number of changes, the number of times the chance of marriage is perceived to decrease. By contrast, mean absolute slope measures the magnitude of both positive and negative changes in the chance of marriage, thus reflecting variability in either direction in commitment to wed, not just negative changes. Because mean absolute slope is not sensitive to differences in absolute levels of commitment (e.g., individuals who report a relatively low and stable rate of commitment over time will have similar scores to those who report a high and stable rate of commitment), it may be a less sensitive indicator of commitment than is proportion of downturns. Future researchers should be sensitive to the idea that commitment and how it changes over time may have different predictors, depending on how it is measured, and should attempt to develop measures of commitment that are sensitive to both absolute levels of and variability over time in commitment.

\section{Limitations and suggestions for future research}

Because the goal of the larger study was to obtain a representative sample of the population, the size of the Hispanic sample in this study was relatively small compared with the size of the Anglo sample. If researchers are interested in studying particular ethnic or racial groups, it is important to oversample for minority group members. Furthermore, special efforts need to be made to recruit Hispanics into research studies. Despite the fact that they are not prevalent in the population, other issues could explain their lack of participation. For example, whereas many Hispanics may speak English, some may be embarrassed by their lack of facility with the language and thus may be reluctant to communicate with an Anglo interviewer. Another possibility is that initially, when being recruited, they may not understand what the research is about and, for fear of being exploited, may decline to participate. Future researchers should address this language barrier by employing bilingual recruiters and bilingual interviewers.

Although Hispanics in this study were treated as a homogeneous population, diversity exists within this population. For instance, Hispanic populations differ with regard to their national 
origin, generational status in the United States, reasons for immigration to the United States, and urban or rural residence. These demographic differences have been related to factors such as educational attainment and socioeconomic status; it is possible that they could also influence the factors that predict commitment to wed. This sample is composed primarily of Mexican-origin Hispanics who have access to a phone and live in a metropolitan area. Results could be different for other Hispanic groups that are less economically advantaged and that live in rural areas.

Furthermore, future research might address in more detail the processes that contribute to commitment to wed among Hispanics. For example, qualitative research (e.g., in-depth interviews) examining the intimate relationships of Hispanic men and women may reveal the meanings that Hispanic men and women assign to the relationship variables measured in these quantitative analyses. Such research also may help elucidate the meaning of other processes that we can only speculate about, such as acculturation and the possible negative repercussions of having overinvolved family members who are attempting to be supportive.

\section{Acknowledgments}

This study was supported by a grant to Catherine A. Surra from the National Institute of Mental Health (R01 MH47975). The authors would like to thank Catherine Surra for the use of these data, as well as all of the interviewers and other staff members who helped with the project.

\section{References}

Booth, A., \& Edwards, J. (1985). Age at marriage and marital instability. Journal of Marriage and the Family, 47, 67-75.

Braiker, H. B., \& Kelley, H. H. (1979). Conflict in the development of close relationships. In R. L.Burgess \& T. L.Huston (Eds.), Social exchange in developing relationships (pp. 135-168). New York : Academic Press.

Bramlett, M. D., \& Mosher, W. D. (2001). First marriage dissolution, divorce, and remarriage: United States (Advance data from vital and health statistics No. 323, pp. 1-19). Hyattsville, MD : National Center for Health Statistics.

Bryant, C. M., Surra, C. A., \& Swim, T. J. (1996, November). A test of the dyadic withdrawal hypothesis: Do African American, Anglo, and Hispanic couples exhibit similar patterns of social regression? Paper presented at the National Council on Family Relations, Kansas City, MO.

Bumpass, L. L., Martin, T. C., \& Sweet, J. A. (1991). The impact of family background and early marital factors on marital disruption. Journal of Family Issues, 12, 22-42. 
Clarke, S. C. (1995). Advance report of final marriage statistics, 1989 \& 1990, Monthly Vital Statistics Report, 43 (12(S)), 16-17. Retrieved February 18, 2002, from http://www.cdc.gov/nchswww/data/mv43_12s.pdf.

Demaris, A., \& MacDonald, W. (1993). Premarital cohabitation and marital instability: A test of the unconventionality hypothesis. Journal of Marriage and the Family, 55, 399-407.

Domino, G., \& Acosta, A. (1987). The relation of acculturation and values in Mexican Americans. Hispanic Journal of Behavioral Sciences, 9, 131-150.

Eberstein, I. W., \& Frisbie, W. P. (1976). Differences in marital instability among Mexican Americans, Blacks, and Anglos: 1960 and 1970. Social Problems, 23, 609-621.

Falicov, C. J. (2001). The cultural meanings of money. American Behavioral Scientist, 45, 313328.

Freeberg, A. L., \& Stein, C. H. (1996). Felt obligation towards parents in Mexican American and Anglo-American young adults. Journal of Social and Personal Relationships, 13, 457-471.

Golding, J. M., \& Baezconde-Garbanati, L. A. (1990). Ethnicity, culture, and social resources. American Journal of Community Psychology, 18, 465-486.

Griffith, J., \& Villavicencio, S. (1985). Relationships among acculturation, sociodemographic characteristics and social supports in Mexican American adults. Hispanic Journal of Behavioral Sciences, 7, 75-92.

Hofstede, G. (1980). Culture's consequences: International differences in work-related values. Beverly Hills, CA : Sage.

Hofstede, G. (1991). Cultures and organizations: Software of the mind. London : McGraw-Hill.

Hughes, D. K., \& Surra, C. A. (1998). Personal, moral, and structural components of commitment: A test of a full model. Unpublished manuscript, Miami University, Oxford, $\mathrm{OH}$.

Hurtado, A. (1995). Variations, combinations, and evolutions: Latino families in the United States. In R. E.Zambrana (Ed.), Understanding Latino families: Scholarship, policy, and practice (pp. 40-61). Thousand Oaks, CA : Sage.

Huston, T. L., Surra, C. A., Fitzgerald, N. M., \& Cate, R. M. (1981). From courtship to marriage: Mate selection as an interpersonal process. In S.Duck \& R.Gilmour (Eds.), Personal relationships 2: Developing personal relationships (pp. 53-88). New York : Academic Press.

Jacquet, S. E., \& Surra, C. A. (2001). Parental divorce and premarital couples: Commitment and other relationship characteristics. Journal of Marriage and Family, 63, 627-638. 
Johnson, M. P. (1991). Commitment to personal relationships. Advances in Personal Relationships, 3, 117-143.

Keefe, S. E. (1984). Real and ideal extended familism among Mexican Americans and Anglo Americans: On the meaning of “close” family ties. Human Organization, 43, 65-70.

Keefe, S. E., Padilla, A. M., \& Carlos, M. L. (1979). The Mexican-American extended family as an emotional support system. Human Organization, 38, 144-152.

Kelley, H. H. (1983). Love and commitment. In H. H.Kelley, E.Berscheid, A.Christensen, J. H.Harvey, T. L.Huston, G.Levinger, E.McClintock, L. A.Peplau, \& D.Peterson (Eds.), Close relationships (pp. 265-314). New York : W. H. Freemen.

Kenny, D. A. (1988). The analyses of data from two-person relationships. In S. W.Duck (Ed.), Handbook of personal relationships (pp. 57-77). London : Wiley.

Kinnaird, K. L., \& Gerrard, M. (1986). Premarital sexual behavior and attitudes toward marriage and divorce among young women as a function of their mothers' marital status. Journal of Marriage and the Family, 48, 757-765.

Larzalere, R. E., \& Huston, T. L. (1980). The Dyadic Trust Scale: Toward understanding interpersonal trust in close relationships. Journal of Marriage and the Family, 42, 595-604.

Mindel, C. H. (1980). Extended familism among urban Mexican Americans, Anglos, and Blacks. Hispanic Journal of Behavioral Sciences, 2, 21-34.

Murphy, S. (1998). A mile away and a world apart. In J. G.Power \& T.Byrd (Eds.), US-Mexico border health: Issues for regional and migrant populations (pp. 2-23). Thousand Oaks , CA : Sage.

Nock, S. L. (1995). A comparison of marriages and cohabiting relationships. Journal of Family Issues, 16, 53-76.

Roberts, R. E., \& Roberts, C. R. (1982). Marriage, work, and depressive symptoms among Mexican Americans. Hispanic Journal of Behavioral Sciences, 4, 199-221.

Rusbult, C. E. (1983). A longitudinal test of the investment model: The development (and deterioration) of satisfaction and commitment in heterosexual involvements. Journal of Personality and Social Psychology, 45, 101-117.

Staples, R., \& Mirande, A. (1980). Racial and cultural variations among American families: A decennial review of the literature on minority families. Journal of Marriage and the Family, 42, 887-903. 
Surra, C. A., Arrizi, P., \& Asmussen, L. A. (1988). The association between reasons for commitment and the development and outcome of marital relationships. Journal of Social and Personal Relationships, 5, 47-63.

Surra, C. A., \& Hughes, D. K. (1997). Commitment processes and accounts of the development of premarital relationships. Journal of Marriage and the Family, 59, 5-21.

Surra, C. A., Hughes, D. K., \& Jacquet, S. (1999). The development of commitment to marriage: A phenomenological approach. In J. M.Adams \& W. H.Jones (Eds.), The handbook of interpersonal commitment and relationship stability. New York : Plenum.

U.S. Census Bureau. (1990). Hispanic Origin—Universe: Persons 1990 summary tape file 1 (STF 1) - 100 percent data[Data file]. Retrieved October 3, 2002, from http://factfinder.census.gov/servlet/BasicFactsServlet?_lang=en.

Vega, W. A. (1990). Hispanic families in the 1980's: A decade of research. Journal of Marriage and the Family, 52, 1015-1024.

White, L., \& Rogers, S. J. (2000). Economic circumstances and family outcomes: A review of the 1990s. Journal of Marriage and the Family, 62, 1035-1051. 